\title{
Implications on laboratory workload of breast cancer screening
}

\author{
B Green, I W McDicken, L S Turnbull
}

\begin{abstract}
Aims: To determine whether the recommended methods for the handling of breast excision biopsy specimens from screening patients cause more laboratory work than those used for non-screening patients.

Methods: All breast excision biopsy specimens from 1990 were identified. Ninety one came from patients identified during the prevalent round of breast screening. These were compared with 127 from non-screening patients operated on by the same surgeon. The workload in terms of initial blocks taken, cases which needed extra blocks or other additional work, and the time taken for delivery of slides to the pathologist were assessed.

Results: The screening cases required significantly more initial blocks than the non-screening cases (8.03 v 4.95; $p=0.000001)$. When looking at the malignant diagnoses this difference was maintained (7.74 $v 6.02 ; p=0.00014)$.

Conclusions: Excision biopsy specimens from screening patients require more laboratory work per case than those from non-screening patients. The reasons for these differences lie in the nature of the specimens and their subsequent diagnoses, and in the initial indications for biopsy.
\end{abstract}

With the implementation of the United Kingdom Breast Screening Programme (UKBSP) pathology laboratories can expect an increase in the overall numbers of breast biopsy specimens. Guidelines have been produced on how laboratories should approach the handling of these specimens, ${ }^{12}$ and descriptions of the methods used in individual laboratories have also been published. ${ }^{3}$ Individual laboratories can use these guidelines to produce local protocols for the handling of breast screening biopsy specimens, based on the available local facilities. The Royal Liverpool University Hospital provides the breast screening service for three Forrest units. In 1990 there were 91 breast excision biopsy specimens received in the pathology department as a result of screening. During the same period there were 229 breast excision biopsy specimens removed from non-screening patients. All of the screening specimens and 127 of the non-screening specimens were received from the same surgeon.

This study describes the different methods of handling the two groups and quantifies the effect of these methods on the laboratory workload.

\section{Methods}

All breast excision biopsy specimens received in the department of pathology in 1990 were identified from a search of the records stored on the departmental computer. They were divided into those from non-screening patients and those from screening patients. The laboratory records for each case were checked individually and the following parameters were recorded:

(i) diagnostic category-benign; malignant (invasive, in situ, both, secondary);

(ii) initial blocks sampled per case;

(iii) additional blocks sampled per case;

(iv) extra work other than extra blocks per case (special stains);

(v) time in working days taken from slides to reach the pathologist with the day of surgery taken as day 0 .

The method of sampling the two groups was different and will be described below. From August 1990 the method for sampling the screening biopsy specimens was also changed to allow for more extensive specimen radiography.

Statistical analysis was performed using Arcus software (IE Buchan, Medical Computing, Aughton, West Lancs.).

NON-SCREENING (GROUP I)

The specimens are delivered to the pathology department by the routine portering service and arrive either on the day of surgery or the following day. They are all placed in $10 \%$ neutral buffered formalin in the operating department and fixed overnight. The specimen is inspected, measured and described before blocks for histological examination are taken by the duty pathologist according to the clinical details and the macroscopic appearances of the lump. Tissue is then processed to paraffin wax sections and the slides delivered to the duty pathologist. Any remaining tissue is stored in the original container.

\section{SCREENING (GROUP II JANUARY TO JULY)}

All specimens in this group undergo whole specimen radiography using the Phillips Diagnost UM machine (Phillips Medical Systems). This either takes place during the operation, to assess adequacy of excision, in which case the specimen is collected from the radiology department by a member of the pathology department, or following overnight fixation. In each case the specimen is fixed in $10 \%$ neutral buffered formalin. The specimen is inspected, 
measured, and described before blocks for histological examination are taken by the duty pathologist according to the macroscopic appearances of the lump and the location of the radiological abnormality. The site of the blocks taken is indicated on a line drawing of the specimen. Tissue is then processed to paraffin wax sections and delivered to the duty pathologist. All slides are then reviewed by the designated breast screening pathologist (IWM) for the UKBSP. Any remaining tissue is stored in the original container.

SCREENING (GROUP III AUGUST TO DECEMBER)

All specimens in this group undergo whole specimen radiography. This may take place during the operation, as described above, or following overnight fixation. Specimens that do not require intraoperative radiography are usually sent to the pathology department unfixed via the Lampson tubes. They are then inspected by the designated pathologist (BG) before they are fixed in $10 \%$ neutral buffered formalin. Only if the specimen is not intact is it further handled at this stage. Any specimen that has been partially incised has the incision extended to bisect the specimen before it is fixed. After overnight fixation the specimen is inspected, measured, and described. If intact the whole surface of the specimen is dried and painted with India ink before being cut into 6-8 $\mathrm{mm}$ thick slices. These slices are placed in a plastic bag, given a number, and then undergo radiography (as above). Blocks for histological examination are taken by the designated pathologist according to the macroscopic appearances and the location of the radiological abnormality. The site of the blocks taken is indicated on the film of the slice radiograph. Tissue is postfixed in Carnoy's fluid (60\% ethanol, $30 \%$ chloroform, $10 \%$ glacial acetic acid) for five hours before processing to paraffin wax sections and delivery to the desigr ted breast screening pathologist (IWM or LT). Any remaining tissue is placed in individual plastic bags labelled with the patient's name and the slice number.

\section{Results}

In 1990 the department received 127 breast excision biopsy specimens from non-screening patients and 91 from screening patients (all operated on by the same surgeon). The breakdown according to the diagnostic category is shown in the table. The ratio of benign:malignant for group I is 1.39 , for groups II and III, and for groups II and III combined, 0.57 . In group I 57 cases $(44.8 \%)$ had prenperative fine needle aspiration cytology (FNAC) and eight $(6.3 \%)$ Tru-cut biopsy performed. In group II $38(80.8 \%)$ had FNAC and one $(2 \cdot 1 \%)$ Trucut biopsy. In group III $43(97 \cdot 7 \%)$ had FNAC.

In group I a total of 629 initial blocks were taken with a mean of 4.95 per case and a range of 1-19. In groups II and III combined there was a total of 731 initial blocks taken with a mean of 8.03 per case and a range of 1-17. (Using the Mann-Whitney test, $p=0.000001$ with $95 \%$ confidence interval of 3 to 4 .)
Diagnostic categories

\begin{tabular}{|c|c|c|c|c|}
\hline & $\begin{array}{l}\text { Group } \\
\text { I }\end{array}$ & $\begin{array}{l}\text { Groups } \\
\text { II and } \\
\text { III }\end{array}$ & $\begin{array}{l}\text { Group } \\
\text { II }\end{array}$ & $\begin{array}{l}\text { Group } \\
\text { III }\end{array}$ \\
\hline $\begin{array}{l}\text { No of cases } \\
\text { Benign } \\
\text { Malignant } \\
\text { Invasive } \\
\text { In situ } \\
\text { Both } \\
\text { Secondary } \\
\text { Benign/malignant }\end{array}$ & $\begin{array}{c}127 \\
74 \\
53 \\
26 \\
6 \\
19 \\
2 \\
1 \cdot 39\end{array}$ & $\begin{array}{l}91 \\
33 \\
58 \\
8 \\
17 \\
33 \\
0 \\
0.57\end{array}$ & $\begin{array}{l}47 \\
17 \\
30 \\
5 \\
7 \\
18 \\
0 \\
0 \cdot 57\end{array}$ & $\begin{array}{l}44 \\
16 \\
28 \\
3 \\
10 \\
15 \\
0 \\
0.57\end{array}$ \\
\hline
\end{tabular}

In group I six cases ( $4 \cdot 7 \%$ ) needed extra blocks and 11 cases $(8 \cdot 7 \%)$ needed extra work. In groups II and III together 11 cases $(12 \cdot 1 \%)$ needed extra blocks and $13(14.3 \%)$ needed extra work.

In group I 121 cases (95.3\%) had slides delivered to the pathologist on day 2 , and six cases $(4.7 \%)$ on day 3 . In groups II and III together 75 cases $(82.4 \%)$ had slides delivered on day 2 and 16 cases $(17.6 \%)$ on day 3 .

To assess the effect of the two different methods of handling the screening specimens groups II and III were compared. In group II there were a total of 365 initial blocks with a mean of 7.76 per case and a range of 3-17. In group III there were a total of 366 initial blocks with a mean of 8.31 per case and a range of 1-16. (Using the Mann-Whitney test, $p=0.15$ with $95 \%$ confidence intervals of 0 to 2). In group II 42 cases $(89 \cdot 3 \%)$ had slides delivered on day 2 and five cases $(10.7 \%)$ on day 3 . In group III 33 cases $(75 \%)$ had slides delivered on day 2 and 11 cases $(25 \%)$ on day 3 .

We also compared the malignant cases from the non-screening and the screening groups. There were 51 primary malignant neoplasms in the non-screening group and 58 in the combined screening groups. There were 307 initial blocks from the non-screening malignancies with a mean of 6.02 per case and a range of 319. There were 449 initial blocks from the screening malignancies with a mean of 7.74 per case and a range of 3-16. (Using the MannWhitney test $\mathrm{p}=0.00014$ with $95 \%$ confidence intervals of -3 to -1 .)

\section{Discussion}

This study has concentrated on the workload implications due to the differences in handling screening and non-screening breast excision biopsy specimens. As the ratio of benign:malignant diagnoses in the screening groups is low at 0.57 it is reasonable to assume that our increase in actual numbers of breast biopsy specimens would be small in relation to the total number of patients screened. ${ }^{4}$

Our figures show that there was an increase in the number of initial blocks sampled from the screening group. Our mean number of initial blocks per case at 8.03 compares with the minimum of six blocks recommended by the Department of Health and Royal College of Pathologists' guidelines, ${ }^{1}$ made up of three blocks from the tumour and at least three from adjacent normal tissue. Despite this mean there were 22 cases in which 10 or more initial blocks were taken, which emphasises the point made 
by Elston and Ellis ${ }^{4}$ concerning comparisons with single block specimens. This figure also compares well with the conclusions of a study looking at the minimum number of blocks needed to avoid missing clinically important lesions in macroscopically benign breast lumps ${ }^{5}$ which suggested that 10 blocks from non-fatty areas would identify all clinically important lesions.

Screening cases might be expected to require fewer than this number as the sampling of biopsy specimens relies not only on the macroscopic appearances but on detailed radiographic findings. However, as a much larger proportion of the screening biopsy specimens were performed for malignant disease (benign: malignant ratio 0.57 for screening cases, compared with 1.39 for non-screening cases), the excess of malignant lesions in this category will undoubtedly have affected the overall sampling rate.

Because of the substantially different benign:malignant ratios, we decided to compare the malignant cases only from the two groups. Again there were significantly more initial blocks taken from the malignancies detected by screening than from the nonscreening cases. This can be explained by the increased proportion of pure in situ disease in the screening group which is often multifocal, poorly defined grossly, and requires much wider sampling for adequate assessment. In situ disease appeared much less frequently in the non-screening group- $-47 \cdot 2 \%$ against $86.2 \%$. Although this may have been due to the disease being at a later stage in its natural history, insufficient tissue might have been sampled distant from any obvious macroscopic abnormality.

When assessing the amount of extra work needed in both groups, although the overall numbers are small, the screening group had roughly double the incidence of the nonscreening group. This may be explained by the screening cases having a higher proportion of very small lesions that may have been missed with the initial sampling, and also a higher proportion of cases with diagnostic difficulties. In addition, we hope that the provision of a dedicated specimen $x$ ray cabinet will improve the currently suboptimal specimen radiography and could reduce the number of extra blocks taken. Although different pathologists were responsible for the decision to take extra blocks, we feel such decision making within the department is uniform.

The rate of preoperative FNAC was roughly double that in the screening compared with the non-screening group. The results of cytological examination in the screening patients are part of the total information required to determine overall management, which may or may not include surgery. Therefore, we feel that this has had little effect on initial histological sampling of subsequent excision biopsy specimens which is based on the gross appearances of the tissue and/or the specimen radiograph. If a lesion is not identified which would account for the cytological findings, then the case is reviewed by the multidisciplinary breast screening team and additional blocks may be sampled. This has occurred in only four of the 11 cases which required extra blocks to be taken.

Although the methods used in handling the screening and non-screening specimens are quite different, the method used for the screening cases caused only a minimal delay in the proportion of slides reaching the pathologist by day 2 . It must be emphasised, however, that the second method of handling the screening specimens relies on a dedicated pathologist and technician, and also on close cooperation with the breast screening unit. This method is time consuming for the individuals concerned. These disadvantages are outweighed by the advantages of a system which permits a much greater degree of correlation between the radiographic and pathological appearances.

This study has shown that the methods recommended for the processing of breast excision biopsy specimens from screening patients led to an increased number of blocks being taken compared with non-screening patients. This increase is maintained when a comparison is made between only the malignant cases. Despite the more complicated procedures used with the screening cases there is no unnecessary delay in the slides reaching the pathologist, and the use of specimen and slice radiography permits precise correlation between the clinical, radiographic, and pathological lesion. This raises the question as to whether all breast excision biopsy specimens from non-screening patients in whom malignancy is suspected should be handled in a similar way to those from screening patients. Perhaps the excess workload in terms of blocks taken and time spent on tissue sampling would be offset by increased diagnostic and prognostic information.

We thank Mr I E Buchan for statistical advice.

1 Sloan JP and members of the Royal College of Pathologists' Working Group. National Health Service breast screening programme: pathology reporting in breast cancer screening. programme: pathology reporting in breast
Oxford: Screening Publications, 1990.

2 Armstrong JS, Davies JD. Laboratory handling of impalpable breast lesions: A review. J Clin Pathol 1991;44:89-93.

3 Anderson TJ. Breast cancer screening: principles and practicalities for histopathologists. Recent advances in histopathology. 14. Edinburgh: Churchill Livingstone, 1989

4 Elston CW, Ellis IO. Pathology and breast screening. Histopathology 1990;16:109-18.

5 Schnitt SJ, Wang HH. Histologic sampling of grossly benign breast biopsies. How much is enough? Am J Surg Pathol 1989;13:505-12. 